In this cohort, $43 \%$ of people were given a humidifier.

There were no statistically significant correlations of any of the variables with humidifier outcome. Chi squared analysis showed no significant difference in the proportion of those people with humidifier versus those without for any of the questionnaire categories.

Conclusion It does not appear to be possible to prospectively predict which patients will require a humidifier with their CPAP. Current practice of symptom-led humidification appears valid.

\section{P255 IMPROVEMENT OF SLEEP APNOEA SEVERITY IN OBESE PATIENTS PRE AND POST BARIATRIC SURGERY-IS THERE MORE TO IT?}

${ }^{1} \mathrm{~N}$ Farah, ${ }^{1} \mathrm{R}$ Gorrela, ${ }^{1} \mathrm{~A}$ Bhowmik, ${ }^{1} \mathrm{GH}$ Bothamley, ${ }^{1} \mathrm{M}$ Menon, ${ }^{2} \mathrm{~K}$ Devalia, ${ }^{2} \mathrm{Y}$ Koak, ${ }^{2} \mathrm{~S}$ Agrawal, ${ }^{2} \mathrm{~K}$ Mannur, ${ }^{1} \mathrm{~K}$ Rajakulasingam; ${ }^{1}$ Department of Respiratory Medicine and Allergy, Homerton University Hospital NHS Foundation Trust, London, UK; ${ }^{2}$ Department of Bariatric Services, Homerton University Hospital NHS Trust, London, UK

\subsection{6/thoraxjnl-2013-204457.407}

Background Obstructive Sleep Apnoea (OSA) is prevalent in obese patients and has substantial impact on health and society ${ }^{1}$. We undertook this analysis to examine changes in Sleep Apnoea severity by studying the Apnoea-Hypopnoea Index (AHI) pre and post bariatric surgery in a London District General Hospital. Methods We retrospectively reviewed patients with a history of OSA who underwent a laparoscopic bariatric procedure between 2011 and 2012, preceded by a sleep study. Repeat sleep studies were performed in those patients who reported significant symptom reduction as part of the assessment to withdraw CPAP therapy. Following this, data concerning changes in BMI and corresponding AHI values were analysed as were Epworth Sleepiness Scale scores. Results Twenty patients reported significant improvement in sleep apnoea symptoms and requested to come off CPAP therapy. Nineteen of them underwent a repeat sleep study. The mean age of the patients was 45 years $($ SEM $=2.1$ ) with 14 of them being females. The mean pre and post-surgery BMI were $49 \mathrm{~kg} / \mathrm{m}^{2}(\mathrm{SEM}=1.4)$ and $40 \mathrm{~kg} / \mathrm{m}^{2}(\mathrm{SEM}=1.8)$ respectively ( $\mathrm{p}=0.000$, paired $\mathrm{t}$-test). The mean Epworth sleepiness scale scores were 13.3 and 7.4 ( $\mathrm{p}=0.001$, paired t test) respectively for the same patients. The average time period for repeat sleep studies was 7 months (range 2 - 19). The mean baseline AHI pre surgery was 41 (SEM $=6.5)$ and the corresponding value postsurgery was $12.4(\mathrm{SEM}=2.7)(\mathrm{p}=0.000$, paired $\mathrm{t}$-test $)$. The correlation co-efficient corresponding to percentage decrease in BMI and AHI was 0.44 (Spearman's correlation). The majority of patients included in this analysis discontinued the use of CPAP and continue to remain well.

Conclusion This analysis demonstrates the positive outcome on AHI and Epworth sleepiness scale scores following laparoscopic bariatric surgery. The correlation between percentage changes in BMI and AHI suggest there may be factors other than weight reduction alone contributing to the outcome.

${ }^{1}$ Scottish Intercollegiate Guidelines Network (2003). Management of Obstructive Sleep Apnoea/Hypopnoea Syndrome in Adults:73. Edinburgh: Scottish Intercollegiate Guidelines Network.

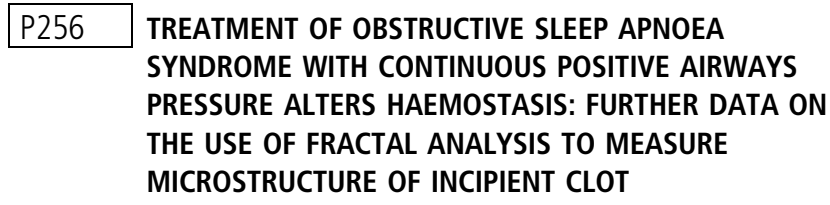

${ }^{1} \mathrm{M}$ Wilczynska, ${ }^{1} \mathrm{KE}$ Lewis, ${ }^{2} \mathrm{~S}$ Stanford, ${ }^{2} \mathrm{M}$ Lawrence, ${ }^{3} \mathrm{~K}$ Hawkins, ${ }^{3} \mathrm{PR}$ Williams, ${ }^{2}$ PA Evans; ' $P$ Prince Philip Hospital, Llanelli, United Kingdom; ${ }^{2}$ NISCHR Haemostasis Biomedical Research Unit, Swansea, United Kingdom; ${ }^{3}$ College of Engineering, Swansea University, Swansea, United Kingdom

\subsection{6/thoraxjnl-2013-204457.408}

Introduction Untreated obstructive sleep apnoea syndrome (OSAS) increases cardiovascular risk and altered haemostasis is at least partly implicated. As previously reported using fractal analysis and a new biomarker called fractal dimension (Df) it is possible to assess the microstructure of incipient clot in whole blood (1). Df relates to the kinetics of clot formation and quantifies clot fibrin network microstructure as it forms. A higher Df represents a more pro-coagulable state. Healthy volunteers have a reproducible Df of 1.74(0.07) (2).

Aim To see if Df changes in OSAS after an acute and subacute treatment with CPAP.

Methods 36 patients with newly diagnosed OSAS: 32 males, mean (SD), BMI = $37.1(7.5) \mathrm{kg} / \mathrm{m}^{2}$, age $56.6(10.2)$ years, $4 \%$ desaturation rate $(4 \% \mathrm{DR})=44.6$ (31.1) events/hour, Epworth Sleepiness Score (ESS) 13.23 (5.0). Blood was collected at baseline prior to CPAP treatment and then after the first night and 4 weeks of CPAP. Samples were tested for fractal analysis (AR-G2 Rheometer, TA Instruments, UK).

Results Patients who were commenced on CPAP were followed up within an average of 36.97(6.29) days. CPAP compliance was overall satisfactory with a mask on average time of 4.43 (1.8) hrs/ night. Repeated overnight pulse oximetry while on CPAP showed a significant improvement in sleep study variables $(4 \% \mathrm{DR}=7.58$ (8.3) events/hr; $\mathrm{p}<0.001$ ) when compared to pre-CPAP measurements. CPAP use resulted in the significant reduction in Df (ANOVA $\mathrm{p}<0.001$ ) (Figure 1). Post-hoc analysis (Tukey HSD) showed that an acute $(1$ night, $\mathrm{p}<0.001)$ and a short period $(1$ month, $p=0.01$ ) CPAP treatment both resulted in the significant change in Df levels when compared to the baseline.

Conclusion As reported previously OSAS is associated with a significantly increased prothrombotic state in the morning that is detected by Df. Acute CPAP use in OSAS is sufficient to alter fibrin clot microstructure which can be quantified with Df. This preliminary data suggest Df could be used as a new sensitive biomarker to assess vascular risk.

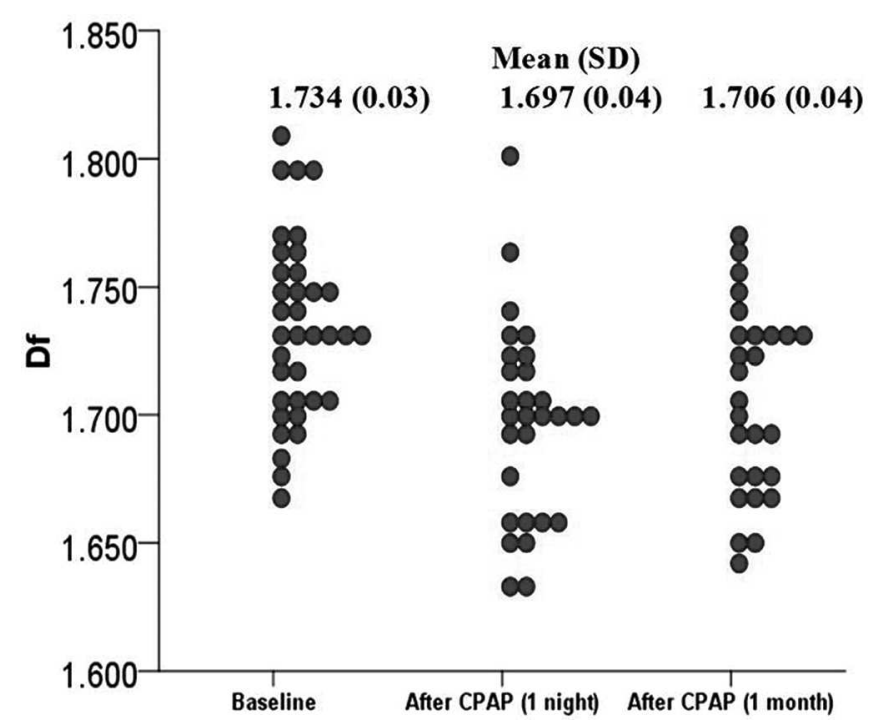

Abstract P256 Figure 1. Individual Df measurements at the baseline and after a night of CPAP treatment and a month of CPAP treatment in OSAS group. 


\section{REFERENCES}

1. Wilczynska M et al Thorax. 2012;67:A23-A23

2. Evans PA et al Blood. 2010;116:3341-3346

\section{P257 SUSTAINED ASPIRIN EFFECTS ON PLATELETS FUNCTION OVER 24 HOURS IN PATIENTS WITH UNTREATED OBSTRUCTIVE SLEEP APNOEA SYNDROME (OSAS)}

${ }^{1} \mathrm{M}$ Wilczynska, ${ }^{1} \mathrm{KE}$ Lewis, ${ }^{2} \mathrm{PR}$ Williams, ${ }^{3} \mathrm{M}$ Lawrence, ${ }^{2} \mathrm{~K}$ Hawkins, ${ }^{3} \mathrm{~S}$ Stanford, ${ }^{3}$ PA Evans; 'Prince Philip Hospital, Llanelli, United Kingdom; ${ }^{2}$ College of Engineering Swansea University, Swansea, United Kingdom; ${ }^{3}$ NISCHR Haemostasis Biomedical Research Unit, Swansea, United Kingdom

\subsection{6/thoraxjnl-2013-204457.409}

Introduction Prevalence of OSAS in people with cardiovascular disease is much higher than in the general population. Many OSAS patients are prescribed Aspirin for secondary prevention of cardiovascular events but the effects of morning Aspirin might be attenuated by night of recurrent apnoeas and intermittent hypoxia. Therefore evening dosing might be more appropriate. Aim To assess platelets function in the afternoon and immediately post-sleep in untreated OSAS subjects who are on long term once daily $75 \mathrm{mg}$ Aspirin (am).

Methods 11 subjects with newly diagnosed and untreated severe OSAS prescribed Aspirin by their physicians: 10 males, (mean + SD) BMI $40.8 \pm 7.2 \mathrm{~kg} / \mathrm{m}^{2}$, age $60.8 \pm 10.02$ years, $4 \%$ Desaturation rate $(4 \%$ DR) $58.4 \pm 40.8$ events/hour.

Platelet aggregation was induced in vitro by collagen (COL), adenosine-diphoshate (ADP), and arachidonic acid (ASPI). Platelet activation was measured by multiple electrode platelet aggregometry $\left(\right.$ Multiplate $\left.{ }^{\mathrm{TM}}\right)$. Blood samples were collected at $4 \mathrm{pm}$ then 7:30am the following morning, prior to the Aspirin dose.

Results Platelet aggregation in response to ASPI was reduced in the presence of Aspirin as expected and the effects were the same in the afternoon (38.6 \pm 44.4 units) versus morning (39.2 \pm 36.2 units).

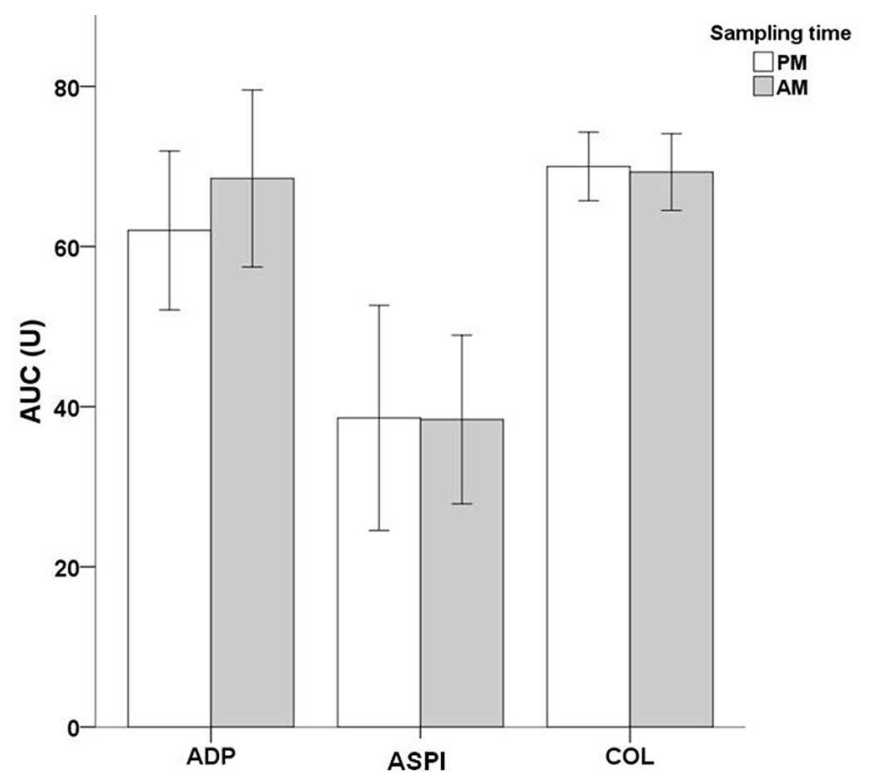

Abstract P257 Figure 1. Platelets aggregation in vitro in response to agonists stimulation: collagen (COL). adenosine-diphoshate (ADP). and arachidonic acid (ASPI) in subjects with severe OSAS on regular morning Aspirin. Results are presented as mean \pm SEM.
There was no difference between afternoon and morning platelet function ( $p>0.05$ for all measures of platelet aggregation using three agonists) (Figure 1).

Conclusions $75 \mathrm{mg}$ of once daily (morning) Aspirin was sufficient to block in vitro platelet aggregation in untreated severe OSAS over a 24 hour period and was not influenced by recurrent apnoeas occurring just prior to blood sampling.

\section{P258 A CASE-CONTROL STUDY OF ASSOCIATIONS BETWEEN HEREDITARY HAEMORRHAGIC TELANGIECTASIA AND COMMON RESPIRATORY CONDITIONS}

FN Abdulla, I Awan, BM Silva, HL Devlin, AE Hosman, CL Shovlin; Imperial College London, London, UK

\subsection{6/thoraxjnl-2013-204457.410}

Background Hereditary haemorrhagic telangiectasia (HHT) is an inherited disease of the vasculature presenting with nosebleeds, telangiectasia and arteriovenous malformations (AVMs). HHT is caused by gene mutations affecting the transforming growth factor beta (TGF- $\beta$ ) superfamily signalling pathway. As TGF- $\beta$ signalling is crucial for embryonic lung maturation, respiratory homeostasis and plays a pivotal role in the pathogenesis of several respiratory diseases, we hypothesised that HHT could alter the respiratory environment and respiratory disease susceptibility. As a first step, we aimed to examine associations between HHT and other respiratory conditions common in the British population.

Methods An online questionnaire at www.imperial.ac.uk/medicine/HHTsurvey2012 was used to collect data. Questions regarding HHT were used to assign HHT status. Participants were categorised based on self-reported status, positive/negative family history and presence of Curaçao criteria (nosebleeds, telangiectasia in specific patterns, and AVMs) Participants' demographics and coexisting respiratory conditions were analysed with Spearman's, Mann-Whitney, and logistic regression analyses using STATA (version 12, Texas USA).

Results By data download on 5 April 2013, 1,465 participants had completed the questionnaire. 1,080 were classified as HHT patients and 179 as controls. Ages ranged from 17-92 (53)years. A history of smoking was provided by $31.5 \%$ of the HHT population and $33.1 \%$ of the controls. In univariate analyses, COPD, venous thromboemboli, pulmonary hypertension and sleep apnoea were more common in the HHT population in comparison to the controls (respectively, $\mathrm{p}=0.038 ; \mathrm{p}=0.019$; $p=0.004 ; p=0.015)$. When multiple regression analyses were used to correct for age and smoking, the association with COPD was lost. However, the positive associations with pulmonary hypertension $(p=0.034)$, venous thromboemboli $(p=0.036)$ and sleep apnoea $(p=0.033)$ remained. Sleep apnoea demonstrated a 2.6 fold higher prevalence in the study HHT population $(69 / 100,000)$ in comparison to published figures for the general population $(27 / 100,000$ from the Hospital Episodes System (HES).

Conclusions The study identified two known associations (HHT and pulmonary hypertension; HHT and venous thromboemboli), supporting the validity of the study methodology. Further investigation is needed to validate and understand the rationale for a possible novel association between HHT and sleep apnoea.

\section{REFERENCES}

1. Lung and Asthma Information Agency. Obstructive Sleep Apnoea. Available at http://www.laia.ac.uk/pdfs/osa.pdf 